\title{
Compton Scattering of Cs-137 Gamma Rays
}

\author{
James E. Parks and Christine P. Cheney \\ Department of Physics and Astronomy, The University of Tennessee, 401 Nielsen Physics Building, Knoxville, \\ TN 37996-1200
}

\begin{abstract}
The Compton Effect ${ }^{1}$ is an ideal physics experiment for the advanced modern physics lab. The relevance of this experiment and an approach to teaching about this lab are discussed. The apparatus for this experiment consists of a Cs-137 gamma source located in a lead howitzer, a goniometer to precisely locate a NaI detector at different angles to make gamma energy measurements, and a multichannel analyzer system that includes a high voltage supply and amplifiers. The lead howitzer and goniometer were specially constructed, and information to reproduce them is given. Typical results are presented.
\end{abstract}

Keywords: Compton Effect, Nuclear, Gamma rays, Radiation, Photoelectric Effect. PACS: 29.30.Kv, 25.20.Dc, 29.40.Mc.

\section{INTRODUCTION}

The scattering of cesium-137 gamma rays by the Compton Effect can be considered the epitome of the ideal advanced modern physics laboratory experiment. It has all the desirable ingredients that are thought to make up the anatomy of a perfect advanced modern physics laboratory experiment. It comprises these essential features:

1. The physics is of the abstract, unseen variety, observable only through instrumentation and is on the microscopic, quantum mechanical, atomic or nuclear scale.

2. The subject has a theoretical model with a basis for investigation.

3. The experiment is an actual experiment and involves the use of preferably new, modern instrumentation and is not a simulation.

4. The experiment yields sufficient data for analysis and verification of the theoretical model.

5. The experiment yields enough data for statistical and error analysis.

6. The experiment is challenging and thought provoking for experimental improvements and analysis of subtle sources of error.

7. Finally, the experiment gives a positive answer to the "DeSerio question:" "Where's the physics in this experiment?"

Compton scattering is a process that can only be observed with instrumentation and measurements based on an abstract, theoretical model. The Compton Effect is a well-established theory that can be verified with analysis of the data. The theory is based on the laws of conservation of energy and momentum being applied using relativistic mechanics. The theory and analysis yield the rest energy of the electron, making it truly a modern physics experiment. The process being studied is on the microscopic level or less. On an atomic scale, the interaction of radiation with matter is studied and applied to the understanding of the detection process and the measurements being made.

In this experiment, scattering and background spectra are measured at various scattering angles to verify the Compton scattering theory. It is an important entry point for studying the interaction of radiation with matter and learning about radiation detectors, energy analysis, and relativistic measurements. It is important for the understanding of basic radiation measurements and sources of background radiation in nuclear physics experiments. Skills acquired in performing experiments are: (1) calibration and use of a multichannel analyzer, (2) use of an apparatus to measure the Compton scattering of gamma rays from a Cs-137 source, and (3) analysis of results to determine the rest mass of the electron. Radiation safety is taught and safe practices are followed.

The detection and measurement of the energy of scattered gamma rays involves the fundamental understanding of the interaction of radiation with matter. These fundamental interactions, described in every introductory modern physics text, can be observed and interpreted with the theory. For example, the photoelectric effect, the Compton Effect, pair production, $\mathrm{x}$-rays, and radiation decay schemes can be studied and used to interpret the data and to extract the relevant energy information to apply in the analysis. A comprehension of how matter interacts with radiation is also necessary to understand how radiation interacts with detectors and is measured. Oddly enough, detection of gamma rays with nuclear detectors and instrumentation involves the same interactions taking place in the detector as those taking place in the

2015 BFY Proceedings, edited by Eblen-Zayas, Behringer, and Kozminski; Peer-reviewed, doi:10.1119/bfy.2015.pr.019

Published by the American Association of Physics Teachers under a Creative Commons Attribution 3.0 license.

Further distribution must maintain attribution to the article's authors, title, proceedings citation, and DOI. 
scattering material being used for the study. In order to understand the measurements and data collected, one must understand how gamma rays interact with the detector.

The experimental data collection involves several variations of the independent variable and each spectrum requires analysis of the data and includes background subtraction to minimize noise and error. Energy spectra are collected and involve an understanding of nuclear physics as well as atomic physics. Not only is the nuclear decay process relevant to this experiment, but the atomic processes involving the interaction of charged particles with matter to produce light are also important.

As one might expect, there are several subtle ways that errors affect measurements. Students can be made aware of these sources of error and their effect on the measurements. Some errors can be dealt with statistically while others have to be minimized. Understanding the errors, as well as their sources and influence on the measurements, is as important as the measurement and theory.

While the use of equipment and the understanding of instrumentation are important in an advanced laboratory, it is more important to teach physics ideas and concepts. So in the end, the "DeSerio" question of "Where's the physics in this experiment?" must have a positive answer. It is fun to work with equipment and to get it to operate, but for an advanced modern physics experiment, it should be used to study physics fundamentals and concepts.

\section{UNDERSTANDING THE INTERACTION OF RADIATION WITH MATTER}

A key role that the Compton scattering experiment provides is promoting the practical understanding of the interaction of radiation with matter. Every textbook lists three important mechanisms through which gamma rays may interact with matter: the photoelectric effect, the Compton Effect, and pair production. Lesser attention is given to $\mathrm{x}$-ray production and bremsstrahlung. These are usually introduced in some abstraction, without practical aspects. For example, the photoelectric effect is discussed, but little is said of it taking place in a sodium iodide crystal used with a photomultiplier tube to detect gamma rays. Numbers and relative magnitudes are usually not discussed. With the photoelectric effect, a Cs-137 gamma ray of $0.662 \mathrm{MeV}$ energy can kick out an electron whose energy is going to be very close to $0.662 \mathrm{MeV}$. A question for the student is, "Is the electron a relativistic electron and does it need to be treated as such?" The next question is, "How far does a $0.662 \mathrm{MeV}$ electron travel in a NaI crystal before coming to a stop?” or "How does a $0.662 \mathrm{MeV}$ electron give up its energy in being stopped in a NaI crystal?" This is where atomic physics becomes important and new questions can be posed to the student. An energetic electron can lose its energy by exciting atoms in the crystal, so "How much energy does it take to excite an atom as it passes by?” The answer of course is a few eV, so the student can be asked about how many atoms are excited. One might also ask, “Suppose, instead of an atom's outer electrons being excited, an inner shell electron is kicked out. How is this different?" This illustrates another purpose of the advanced laboratory experiment, which is to build a knowledge base of magnitudes of different quantities and to develop an intuitive feel of what is taking place. Their sizes, both in absolute and relative scales, are crucial for understanding what is important and what may be neglected. Such thought provoking discussion can be had with students to begin bridging the gap between the purely academic textbook material and the practical world of research and development.

A similar interrogation of students' concepts and understanding can be used to elucidate similar information from them pertaining to the Compton Effect. Questions like, "Under what conditions can a scattered gamma ray impart the most energy to an electron?" or "What range of energies can be given to an electron by a gamma ray?" can be useful. It can be astonishing sometimes how well students know the textbook materials but how little they understand about how it applies to real situations. It is observed that there exist significant differences between the typical introductory physics laboratories and the advanced modern physics labs. Students need help with the transition from the more "cut and dried cookbook" type of labs to more thought provoking, inquiring labs requiring deeper understanding of the subtle, much more esoteric concepts in the modern physics labs. With much attention given to improved pedagogies for teaching physics, this centuries old Socratic method seems to still be a very effective way to introduce modern day experimental methods and measurements. The Compton Effect is an excellent vehicle for this teaching philosophy.

\section{DESCRIPTION OF APPARATUS}

The apparatus for the Compton scattering experiment is shown in Figure 1 and consists of a Cs-137 gamma source inside a shielded, collimator howitzer, a goniometer, a sodium iodide crystal mounted on a photomultiplier tube, a cylindrical aluminum scatterer, and a computer-controlled multichannel analyzer system. Implementation of a Compton scattering experiment with Cs-137 gamma 
rays is complicated by the need for an intense source, license, shielding and collimation, and a means of measuring gamma energies of the scattered rays as a function of the scattering angle. In this instance a 30 millicurie Cs-137 is used for the gamma source. The source is located in a lead howitzer that is used to shield personnel from exposure to radiation and to collimate the beam. The howitzer is not only designed to collimate the gamma rays and provide shielding, but also to be secure and to be turned on and off. The $30 \mathrm{mC}$ source was obtained from GSA Global, formerly AEA Technology. The cesium is rendered in a chemically non-reactive form and then is encapsulated in a welded 4.5 x $6 \mathrm{~mm}$ stainless steel cylinder. The howitzer is made into a 6" diameter lead cylinder approximately 8" long. The source itself is mounted off center in a thick brass disk that can be rotated into position and aligned with the exit channel of the collimator. The brass disk is constrained to rotate inside a stainless steel enclosure located in the middle of the lead howitzer. Spring-loaded balls act as detents in the enclosure so that the source can be precisely rotated into alignment, or out of position for safe storage. A locking pin with a padlock secures the source in the safe position when the source is not in use or is in storage. When the source is in position, gamma rays exit the howitzer via a collimation channel that is simply a 0.25 " diameter hole drilled axially to the howitzer and to the source location.

The goniometer is designed to precisely rotate the detector about the scatterer and to measure the angle of detected scattered gamma rays. An angle scale is engraved to clearly indicate angles of the detector arm. An array of holes is located on the base of the goniometer so that the detector arm can be located at set angular positions with a spring-loaded pin, each hole being $5^{\circ}$ apart. The detector arm supports the NaI photomultiplier detector assembly and a block of lead shielding around it with an aperture to provide directional sensitivity by insuring that the detected gamma rays are emanating from the scatterer. The base of the support arm and the angular scale of its hub use ball bearings to make the heavy components move with ease. The aluminum scatterer is positioned in line with the gamma ray beam and is held in place with an adjustable support rod located in the center of the angular scale.

Gamma ray energy spectra are measured with a Spectrum Techniques UCS30, computer-interfaced, multichannel spectrometer system. The system is completely computer controlled and includes the high voltage supply, pre-amp, and amplifier for the photomultiplier/NaI crystal detector. Software included with the system allows one, two, and three point calibration of energy versus channel number. Also, high voltage and amplifier gain settings are automated.
Data are collected for the energy spectra of scattered gamma rays, and background spectra can be taken and subtracted from the measurements using this built-in feature of the software.

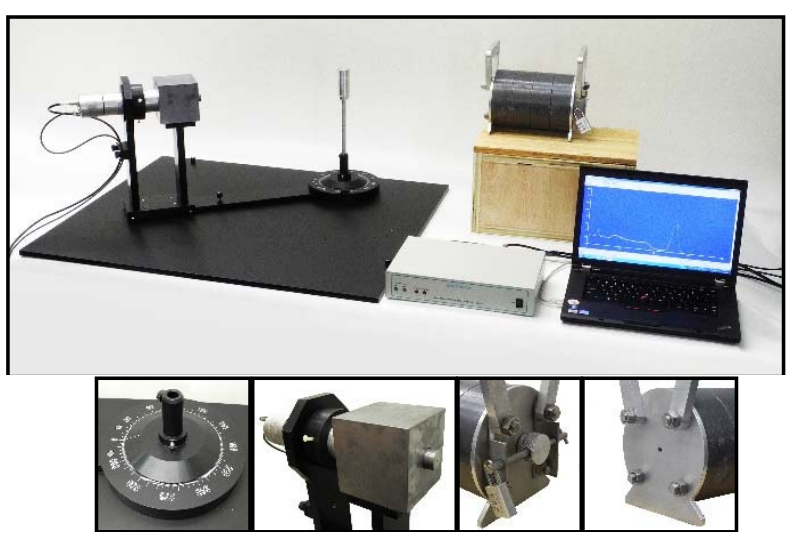

FIGURE 1. The apparatus for the Compton scattering experiment consists of a Cs-137 gamma source inside a shielded, collimator howitzer, a goniometer, a sodium iodide crystal mounted on a photomultiplier tube, a cylindrical aluminum scatterer, and a computer controlled multichannel analyzer system.

\section{PROCEDURE}

The Compton scattering equation is given by

$$
\frac{1}{E^{\prime}}-\frac{1}{E}=\frac{1}{m_{0} c^{2}}[1-\cos \theta]
$$

where $E$ and $E^{\prime}$ are the initial and final energies of the scattered gamma ray, $\theta$ is the scattering angle, and $m_{0} c^{2}$ is the rest mass of the electron. The initial energy of the gamma ray is measured without a scattering object in place and then the final energy is its energy after being scattered by the object. Care has to be taken to limit the count rate for the measurements made without scattering with gammas coming directly from the source. Final energies are typically measured at each angle for angles $10^{\circ}$ to $110^{\circ}$ in increments of $10^{\circ}$. As the angles get larger the count rate for Compton scattered gamma rays becomes smaller and the background becomes increasingly more significant. Therefore, the counting times have to be increased as the angles get larger. To compensate for the background, a measurement is made with the scatterer in place. A background measurement is then made for an equal time with the scatterer removed, leaving everything else the same. The Spectrum Techniques software allows the background spectrum to be stripped from the spectrum with the scatterer in place. The energy of the scattered gamma ray is measured by the position of the photoelectric peak. The software has 
a peak finding function and can find the centroid for points making up the photoelectric peak.

\section{RESULTS}

An energy spectrum for unscattered gamma rays from the source is measured and used for calibration. The photoelectric peak appears at $662 \mathrm{keV}$ as it should after the instrument is calibrated. To illustrate typical Compton scattering, Figure 2 shows an energy spectrum for gamma rays scattered at an angle of $50^{\circ}$ relative to the direction of the gamma rays being emitted from the source. Also included in Figure 2 is a background spectrum taken with the detector set at $50^{\circ}$, but with the scatterer removed. Everything else was unchanged including the measurement time. Figure 3 shows the spectrum with the background stripped and indicates that the photoelectric peak is located at about $440 \mathrm{keV}$ for scattering at $50^{\circ}$. The unscattered spectrum is also included for comparison, but it has been normalized to the height of the $50^{\circ}$ peak by a factor of 15 so that the photoelectric peaks are at about the same height.

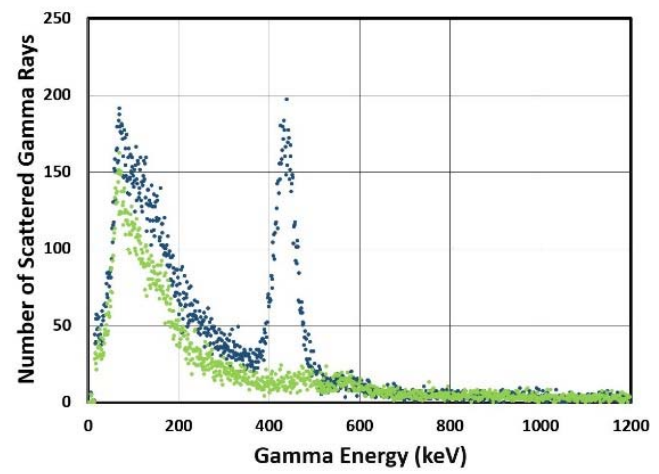

FIGURE 2. A typical energy spectrum for Cs-137 gamma rays scattered at an angle of $50^{\circ}$ relative to the direction of the gamma rays being emitted from the source. Also shown is a background spectrum taken with the detector set at $50^{\circ}$, but with the scatterer removed.

\section{ANALYSIS}

Data taken at the various angles results in a data table of scattered peak energies versus the scattering angles. Values of $\frac{1}{E^{\prime}}-\frac{1}{E}$ are calculated and plotted as a function of $[1-\cos \theta]$, which yields a straight line in accordance with Equation (1). The slope is then the factor $\frac{1}{m_{0} c^{2}}$, and its inverse value is the rest mass of the electron, $m_{0} c^{2}$, a well-known value that can be used for comparison. A typical set of student data is shown graphed in Figure 4 and shows a very linear relationship as it should. However, the slope of this data yields $536 \mathrm{keV}$ for the rest mass and is about $5 \%$ too large for the accepted value of $511 \mathrm{keV}$. This would indicate a systematic error in the experimentperhaps a calibration error. There are a number of subtleties that make for good discussion as to why some discrepancies may occur. ${ }^{2}$

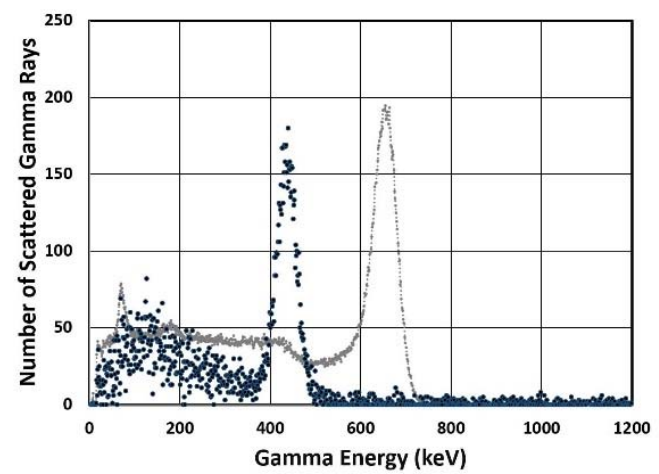

FIGURE 3. Energy spectrum of Compton scattered Cs-137 gamma rays at $50^{\circ}$ with the background stripped. A normalized unscattered spectrum for $0^{\circ}$ is also included for comparison to see the energy shift.

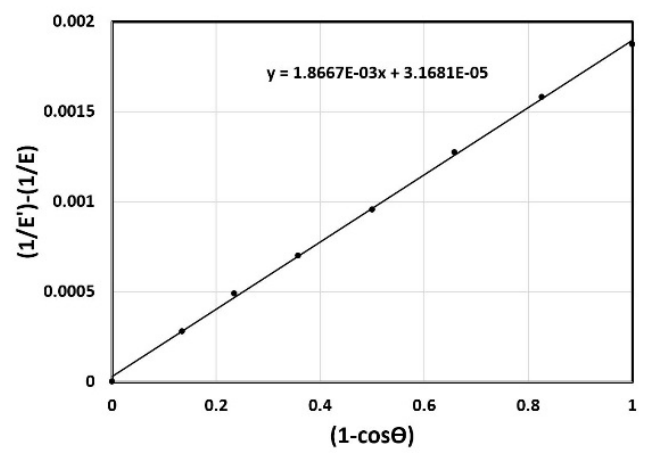

FIGURE 4. A typical set of student data is shown for values of $\frac{1}{E^{\prime}}-\frac{1}{E}$ plotted as a function of $[1-\cos \theta]$.

\section{REFERENCES}

1. Arthur H. Compton, "A Quantum Theory of the Scattering of X-rays by Light Elements," The Physical Review, 21, No. 5, 483-502 (May, 1923).

2. G. F. Knoll, Radiation Detection and Measurement (John Wiley \& Sons, Inc., New York, 2000), pp. 307-342. 